\title{
Fracture-matrix transfer function in fractured porous media
}

\author{
A. J. Mahmood \\ Department of Chemical Industries, Al-Anbar Technical Institute, Iraq
}

\begin{abstract}
One of the mathematical models dealing with fluid flow in fractured porous media is the dual porosity model. The key issue governing this model is the transfer function which representing fracture-matrix interaction. Many formulas for this function had been presented since the 1960s. In this work, a review for these formulations was done. Based on the assumption used in deriving the transfer function formulas, they are classified into two groups. The first group includes those derived assuming semi-steady (pseudo-steady) state flow and the second includes those assuming transient state flow. This work will help worker selecting the formulation that best represent the flow situation and the type of porous media.
\end{abstract}

Keywords: transfer function, fractured porous media, shape factor.

\section{Introduction}

Fluid flow in fractured porous media encountered significant progress which resulting from the efforts and researches conducted to present a mathematical model best representing the phenomena. The key issue in any mathematical model representing fluid flow in a fractured porous media is how to handle the fracture - matrix interaction in different conditions. Two main models were widely used in solving the flow mechanism in fractured porous rock, the first is the dual porosity model presented by Barrenblatt et al. [1] and Warren and Root [2] which considers the highly permeable fractures are entirely responsible for flow between matrix blocks and flow to the well bore while the matrix blocks act as source of fluids.

The second model is the dual porosity dual permeability. This concept may be attributed to Chen Huan-Zhang [3]. It permits matrix-matrix to communicate in addition to matrix-fracture flow. This connection induces capillary continuity 
through the connected matrix block. The mathematical formulations representing the both models include a function named the "transfer function" representing the fracture-matrix interaction. Shape factor is one of the important parameters in calculating the transfer function.

Since the 1960s, many researchers tried to give a mathematical formulation for the transfer function, including the shape factor, that best represent the actual relation between the fracture and the matrix system.

Many workers on the fluid flow in fractured porous media face a dilemma in selecting the proper mathematical formulation for the transfer function; this research presented a review for these formulations to enable workers on fluid flow in naturally fractured porous media selecting the proper one.

\section{The original model}

In 1963 Warren and Root [2] introduced the original dual porosity concept for fractured reservoirs. Their model assumes a continuous uniform fractured network oriented parallel to the principle axes of permeability. The matrix block and the fracture network in this model are occupied the same physical space and assumed identical rectangular parallel piped with no direct communication between matrix blocks. The mathematical model describing 1D flow of slightly compressible fluid for dual porosity model is:

$$
\frac{K_{f}}{\mu} \frac{\partial^{2} P_{f}}{\partial x^{2}}-\tau_{m f}=\emptyset_{f} C_{f} \frac{\partial P_{f}}{\partial t}
$$

Eqn. (1) is similar to the continuity equation for a single porosity model except for the presence of the term $\left(\tau_{m f}\right)$, this term represent the fracture matrix interaction. It has the following formula:

$$
\tau_{m f}=\frac{\sigma K_{m} V}{\mu}\left(P_{f}-P_{m}\right)
$$

The parameter $\sigma$ defined as the shape factor; it reflects the geometry of the matrix and the fracture intensity. For a cubic matrix block $\sigma$ defined as:

$$
\sigma=\frac{2 N(N+2)}{L^{2}}
$$

where $\mathrm{L}$ is the length of the matrix block and $\mathrm{N}$ is the number of normal sets of fractures; 1,2 or 3 . If rock matrix block (cubic) have dimensions a, b, and c, then

$$
\begin{cases}L=a & \text { for } N=1 \\ L=\frac{2 a b}{a+b} & \text { for } N=2 \\ L=\frac{3 a b c}{a b+b c+c a} & \text { for } N=3\end{cases}
$$

\section{Modifications}

There are four principal recovery processes in fractured reservoirs: fluid expansion, capillary imbibition, gravity drainage, and diffusion. These different effects will give the overall transfer between fracture and matrix. 
Workers try to modify the original model by either modifying the original equation of the transfer function to include the different physical interaction or/and presenting modified values for the shape factor only.

This work classifies the modifications into two groups, the first group includes modifications or new relations derived based on the assumption of semi-steady state diffusivity equation while the second group includes that based on transient and semi-steady state diffusivity equation.

\subsection{Group one: pseudo-steady state functions}

The Warren Root formulation was extended for three-dimension multiphase flow by Kazemi et al. [4]. Based on a direct material balance in a cubic matrix block under assumption of pseudo- steady state flow and matrix pressure equals the pressure in the center of the matrix block. They introduce the following transfer function formulation;

$$
\tau_{m f}=\sigma K_{m} \lambda_{\alpha} \rho_{\alpha}\left[\left(P_{\alpha}-\gamma_{\alpha} D\right)_{f}-\left(P_{\propto}-\gamma_{\alpha} D\right)_{m}\right]
$$

They give also a definition for the shape factor as:

$$
\sigma=4\left[\frac{1}{L_{x}^{2}}+\frac{1}{L_{y}^{2}}+\frac{1}{L_{z}^{2}}\right]
$$

Gilman and Kazemi [5] updated the earlier dual porosity simulator of Kazemi et al. [4] by modifying the treatment of mobility. They include the fracture relative permeability in eqn.(5), when fluid is flowing from the fracture into the matrix, so the transfer function calculated as :

$$
\tau_{m f}=\sigma K_{m} \rho_{\alpha}\left\{\omega \lambda_{\alpha m}+(1-\omega) \lambda_{\alpha f}\right\}\left[\left(P_{\alpha}-\gamma_{\alpha} D\right)_{f}-\left(P_{\alpha}-\gamma_{\alpha} D\right)_{m}\right]
$$

where $\omega$ a weight factor is varies from zero to one and equals to one if flow is from the matrix to the fracture.

To include the gravity effects, Thomas et al. [6] introduce the pseudo relative permeability and capillary pressure curves. They use the term $\lambda_{\alpha f}^{*}$ instead of $\lambda_{\alpha f}$ in eqn.(7) and calculate it depending on the flow direction; if water flowing from the fracture to the matrix $k_{r w}$ calculated from:

$$
K_{r w}=S_{w f}\left[K_{r w}\right]_{P c=0}
$$

and if oil flows from the fracture to the matrix system Kro is calculated from

$$
K_{\text {ro }}=S_{o f}\left[K_{\text {ro }}\right]_{S w m}
$$

For flow from the matrix to the fracture, unaltered relative permeability values are used. To include the effect of block coverage they multiply the matrix phase relative permeability value by the fracture phase saturation, i.e. not all matrix block within a computational cell will undergoes imbibitions.

Litvak [7] presented a dual porosity formulation with a modified gravity potential

$$
\tau_{m f}=\sigma K_{m} \rho_{\alpha} \lambda_{\alpha} C_{\alpha f}\left[P_{\alpha f}-P_{\alpha m}-\left(\gamma_{\alpha m}-\gamma_{\alpha f}\right)\left(D_{\alpha m}-D_{\alpha f}\right)\right]
$$


The gravity term in eqn. (10) defines the gravity head with segregated water level in the matrix and fracture. The $\mathrm{C}_{\alpha f} C_{\alpha f}$ multiplier is a coverage factor. For blocks completely immersed in water $\mathrm{C}_{\alpha \mathrm{f}}=1 C_{\alpha f}=1$, meaning that there will be a large imbibition transfer rate than a block partially immersed in water for which $\mathrm{C}_{\alpha \mathrm{f}}<1 C_{\alpha f}<1$.

Sonier et al. [8] presented another modification for eqn. (7) by assuming that the phase saturations within the fracture are the same in any portion of the grid cell and all matrix blocks have the same saturation. For an oil water system, the water level in the fracture and matrix defined as:

$$
\begin{gathered}
D_{f}=\frac{\left(S_{w f}-S_{w i f}\right) L_{z}}{1-S_{\text {orf }}-S_{w i f}} \\
D_{m}=\frac{\left(S_{w m}-S_{w i m}\right) L_{z}}{1-S_{\text {orm }}-S_{\text {wim }}}
\end{gathered}
$$

In 1992, Kazemi et al. [9] performed imbibition experiments and fitting the data into single correlation. They proposed a generalized shape factor as:

$$
\sigma=\frac{1}{V_{m}} \sum_{j=1}^{N}\left(\frac{A_{m}}{d_{m}}\right)_{j}
$$

$A_{m}$ is the area of a surface area open to flow in a given direction and $d_{m}$ is the distance from the open surface to the centre of the matrix block. The summation is done over all open surfaces of the matrix block.

The diffusivity equation for single-phase flow at semi-steady state solved by Coats [10] to give a shape factor of $12 / \mathrm{L}^{2}, 28.45 / \mathrm{L}^{2}$ and $49.58 / \mathrm{L}^{2}$ for the one, two and three-dimensional cubic rock matrix respectively. However, he recommended using $8 \mathrm{~N} / \mathrm{L}^{2}$ for calculating the shape factor.

Lim and Aziz [11] presented the derivation of new shape factor by combining the geometrical aspect of the system with analytical solutions of the pressure diffusion equation for flow between the matrix and the fracture. They found that the shape factor for a system with one set of fracture is $\pi^{2} / \mathrm{L}^{2}$, while the resulting shape factors are $18.17 / \mathrm{L}^{2}$ and $25.67 / \mathrm{L}^{2}$ for two and three sets of fractures respectively.

They also showed that the shape factor for system with anisotropic rectangular matrix block is:

$$
\begin{gathered}
\sigma=\frac{\pi^{2}}{L^{2}}=\frac{9.87}{L^{2}} \sigma=\frac{\pi^{2}}{L^{2}}=\frac{9.87}{L^{2}} \\
\sigma=\pi^{2}\left(\frac{1}{L_{x}^{2}}+\frac{1}{L_{y}^{2}}+\frac{1}{L_{z}^{2}}\right)
\end{gathered}
$$

Yu-Shu et al. [12] presented a physical based upstream weighting scheme for modelling fracture matrix flow and interaction in fractured reservoirs. This approach based on continuity of capillary pressure at the fracture interface in estimating physical correct relative permeability for calculating multiphase flow between them. They derive a relation to calculate the cumulative fracture-matrix mass flow or exchange rate: 


$$
Q_{F M}=\frac{(B / 2)^{2}}{D_{F}} \frac{q_{F M}}{P}
$$

where $q_{F M}$ is the Laplace transformed transient fracture- matrix flux, $B$ the fracture spacing or the dimension, $D_{F}$ diffusivity term for the fracture.

Heinemann and Mittermeir [13] presented a study to show that the shape factor derived by Kazemi et al. [9] is generally valid irrespective of any assumption regarding the shape of the matrix blocks surrounded by the fracture network and the number of phases. They extended eqn. (13) to derive a generalized shape factor for anisotropic matrix permeability to be,

$$
\sigma=\frac{1}{V_{m}} \sum_{j=1}^{N} A_{i j} \frac{\left|\bar{k} \vec{n}_{j}\right|}{\left|d_{j}\right|}
$$

where $i, j$ grid point index, $\overline{\bar{k}}$ permeability tensor,$\vec{n}$ normal unit vector and $d$ is the distance between two grid points.

\subsection{Group two: transient functions}

The assumptions of orthogonal fracture system, one dimensional pseudo steady state flow generally incorporated in deriving a transfer function formula resulted in limitations in these formulations.

Many workers tried to derive a more general formulation by taking into consideration the possible naturally occurred lithology and flow conditions like non-orthogonal fracture system, three-phase flow, transient flow and fully or partially immersed matrix which cause the rise of a new formulation for the shape factor.

Chang [14] derived the shape factor in the transfer function by solving the three-dimensional diffusivity equation of a rock matrix block under unsteadystate production in four cases by assuming different boundary conditions. (1) Constant fracture pressure; (2) constant flow rate; (3) constant fracture pressure followed by linearly declining fracture pressure; and (4) linearly declining fracture pressure followed by constant fracture pressure.

Four different time dependent formulation were presented. For the case of constant fracture pressure and 1-D matrix-fracture flow in the x-direction, the shape factor was:

$$
\sigma=\frac{\pi^{2}}{L_{x}^{2}} \frac{\sum_{m=0}^{\infty} \exp \left[-(2 m+1)^{2} \pi t_{D C}\right]}{\sum_{m=0}^{\infty} \frac{1}{(2 m+1)^{2}} \exp \left[-(2 m+1)^{2} \pi t_{D C}\right]}
$$

where

$$
t_{D C}=\frac{k_{x} t}{L_{x}^{2} \emptyset \mu c}
$$

He concluded that the shape factor values are time-dependent under the unsteady state condition and it decreases with depletion time in the transient stage and converges to a constant value in semi-steady state.

Another approach was by Sarma [15], Sarma and Aziz [16]. They tried to overcome limitations by deriving a final form for the transfer function as: 


$$
q_{w m f}=V \rho_{w} \lambda_{w} \sigma_{P D}\left(P_{w}-P_{w f}\right)-V \emptyset \rho_{w} \sigma_{S D}\left(S_{w}-S_{w c}\right)
$$

For the particular case of two parallel fracture with pseudo-steady state pressure diffusion and instantaneously filled fracture, they define the shape factors in eqn. (19) by

$$
\sigma_{P D}=\frac{\pi^{2}}{L^{2}} \quad \text { and } \quad \sigma_{S D}=b t^{-1}
$$

where $b$ is a constant depending on the porous media properties.

Good match was obtained with a reference solution using a fine grid model with ECLIPES 100 for a conceptual representation of a fractured reservoir with source and sink.

Rangel-German and Kovscek [17] conducted a series of experiments to study air and oil expulsion from rock samples by capillary imbibitions of water in 3D geometry. They reported two different modes of matrix and fracture fill up. Relatively slow flow through fracture is found when fracture to matrix fluid transfer is rapid, fracture aperture is wide, and/or water injection is slow. In this regime, fracture fill slowly with fluid and the regime referred as "filling fracture.” On the other hand, a relatively slower rate of fracture to matrix transfer, narrow apertures, and/or high water injection rates leads to rapid flow through fractures. This regime is labelled "instantly filled". They found that in the first regime the mass of water-imbibed scales linearly with time while in the second one the imbibitions performance scales as the square root of time.

An analytical model was developed to describe the amount of water transfer into the matrix so that matrix-fracture transfer function and the shape factor necessary for numerical simulation can be formulated.

Based on the experimental results Rangel-German and Kovscek [18] perform a dimensional analysis for the experimental data to derive a new time dependent matrix-fracture transfer shape factor formulation and transfer function for both filling and instantly filled fracture transfer.

They presented transfer function relation is similar to that presented by Sarma and Aziz [16] using the same definition for $\sigma_{P D}$ but they gave a new definition for $\sigma_{S D}$ :

$$
\sigma_{S D}=\sigma^{*}\left(\frac{t_{D}}{t_{D}^{*}}\right)^{-m} \quad \text { for } \quad t_{D}<t_{D}^{*}
$$

and

$$
\sigma_{S D}=\sigma^{*} \quad \text { for } \quad t_{D} \geq t_{D}^{*}
$$

where $t_{D}^{*}$ is 0.1 and $m$ is a function of flow rate and fracture aperture. This formulation converges to $\sigma^{*}$ for pseudo-steady state condition.

In 2010, Rangel-German et al. [19] solve the pressure diffusivity equation for single phase using non-uniform boundary conditions and approximating solution with an exponential function. They obtain values for $\sigma_{P D}$ in eqn.(19) for one, two, and three sets of orthogonal fractures, they are $4 \pi^{2} / L^{2} 4 \pi^{2} / L^{2}, 8 \pi^{2} / L^{2}$ and $12 \pi^{2} / L^{2} 8 \pi^{2} / L^{2}$ respectively. 
The approximated shape factors provide good results for systems when the transient behaviour of pressure is short.

Huiyun et al. [20] presented a general formulation for the transfer function. Their formulation is a sum of contributions resulting from the different physical effects, with a functional form that gives the correct early and late-time behaviours:

$$
\begin{aligned}
& \Gamma_{c}=\sum_{p=o, w, g}\left[\Gamma_{p c d}+C_{p c m}\left(T_{p e}+\emptyset_{m} \sum_{q \neq p} T_{p q s}\right)\right] \\
& \tau_{c}=\sum_{p=o, w, g}\left[\tau_{p c d}+C_{p c m}\left(\tau_{p e}+\emptyset_{m} \sum_{q \neq p} \tau_{p q s}\right)\right]
\end{aligned}
$$

where $\Gamma_{\mathrm{c}}$ is the transfer rate of component c. $\tau_{p c d} \Gamma_{\mathrm{pcd}}$ is transfer caused by molecular diffusion; $\mathrm{T}$ is a transfer function with units of rate. $\tau_{p e} \mathrm{~T}_{\mathrm{pe}}$ is the transfer rate of phase $p$ resulting from fluid expansion, $\mathrm{T}_{\mathrm{pqs}}$ is the transfer of phase $p$ resulting from displacement by phase $q$ governed by saturation changes.

They gave detailed mathematical formulas for the components of eqn. (23). These formulas include pressure and saturation dependent parameters.

Hassanzadeh et al. [21] focused their study on single-phase flow between matrix and fracture when the matrix pressure is isotropic. They conducted an analytical solution for the partial differential equation describing flow of slightly compressible fluid in fractured media subjected to three different boundary conditions (constant pressure at matrix border, constant rate at matrix boundary, and constant rate at wellbore) to evaluate a numerical value for the shape factor. A slap shaped (1D), cylindrical-shaped (2D), and spherical shaped (3D) matrix blocks were used with radial and linear flow models. Results show that the value of the shape factor is time dependent at early stages then converges to a stabilize value and the flow model does not effect on the stabilized values of the shape factors. They ranges from $9.87 / \mathrm{L}^{2}$ for the case slab with constant pressure at matrix boundary to $12 / \mathrm{L}^{2}$ for the case of slab with constant rate at wellbore.

Golghaddashti [22] utilized a mathematical model to obtain shape factor for gas-oil gravity drainage mechanism for a single matrix block in a gas cap where matrix block and surrounding fractures fully saturated by oil and gas respectively. He presented a general form to calculate matrix-fracture transfer function in gas-oil system using the following expression for the shape factor:

$$
\sigma L_{Z}^{2}=\frac{\pi^{2}}{4} \frac{\sum_{n=0}^{\infty} \frac{(-1)^{n}}{(2 n+1)} \exp \left(-\left(\frac{2 n+1}{2}\right)^{2} \pi^{2} t_{D}\right)^{2}}{\sum_{n=0}^{\infty} \frac{(-1)^{n}}{(2 n+1)^{3}} \exp \left(-\left(\frac{2 n+1}{2}\right)^{2} \pi^{2} t_{D}\right)}
$$

In this equation, the shape factor starts from the value of (2) and demonstrates a transient behaviour at early time then converges to $\pi^{2} / 4$ at late time. The time variation of the shape factor is negligible after $t_{D}=0.4 t_{D}=0.4$ then it becomes constant after $\mathrm{t}_{\mathrm{D}}=0.7 t_{D}=0.7$.

The obtained model verified against several fine grids numerical simulators as well as two major existing models used in commercial simulators. 
Ranjbar and Hassanzadeh [23] solved the gas diffusivity partial differential equation using the combination of the integral method and the method of moment to derive a shape factor for gas flow in dual porosity system. Accounting for both transient and pseudo steady state fluid exchange between matrix and fracture. They presented the following equation for the late time dimensionless shape factor:

$$
\begin{gathered}
\sigma L_{Z}^{2}=\frac{4 \beta \eta_{D 1}}{\eta_{D}} \frac{1.964 \exp \left(\beta \lambda_{1} \eta_{D}\right)+4.763 \exp \left(\beta \lambda_{2} \eta t_{D}\right)}{0.790 \exp \left(\beta \lambda_{1} t_{D}\right)+0.148 \exp \left(\beta \lambda_{2} t_{D}\right)} \\
\text { for } t_{D} \geq \frac{1}{24 \beta \eta_{D 1}}
\end{gathered}
$$

The parameter $\beta$ is the correction factor that considers the effect of the variability of the hydraulic diffusivity with distance. The other parameter $\eta_{D 1}$ is a function of pressure, specific gravity, and temperature. These two unknown parameters used as matching parameters.

Similar to the flow of slightly compressible fluid, the shape factor demonstrate a transient behaviour then converge to a constant value during the pseudo-steady state.

The average value of the shape factor for compressible gas flow in one set of fractures under constant fracture pressure boundary conditions was 8.5/ $\pi^{\wedge} 28.5 / \pi^{2}$.

Their results reveal that the stabilized value of the shape factor for a compressible fluid is a weak function of temperature, gas specific gravity, and pressure variation.

Ranjbar et al. [24] investigated the effect of the fracture pressure depletion on the shape factor for single-phase flow of a compressible fluid. Three fracture pressure regimes are used. They are constant, linearly declining and exponentially declining fracture pressure. They presented two formulas to calculate the shape factor for each fracture pressure regime, one for the early time (transient) and the second is for the late time. They showed that the shape factor values for all the pressure regimes tend to a stable value. These values vary between an upper limit of 10.38 for linearly declining fracture pressure and a lower limit of 8.57 for constant fracture pressure.

The results of the formulas presented by the two pre-mentioned researches validated with fine grid numerical simulator.

\section{Conclusions}

1. In spite of all efforts, there is no general formula for the transfer function (including the shape factor) which can be considered as a general one.

2. The shape factors derived based on pseudo steady state flow converges to a fixed value after certain time.

3. The proper formulation of the transfer function and shape factor may be selected depending on the reservoir conditions. 


\section{Nomenclature}

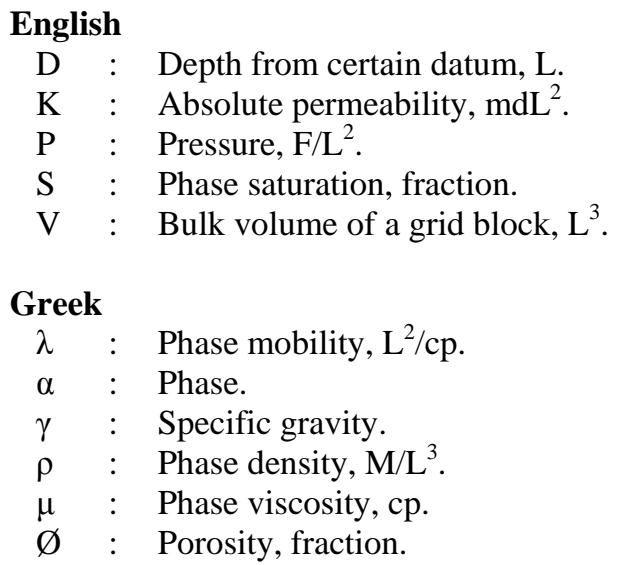

\section{Subscript}

f : Fracture system.

g : Gas.

m : Matrix system.

o : Oil.

w : Water.

* : $\quad$ Variable in Laplace space.

\section{References}

[1] Barrenblatt, G.J., Zheltov, I. P., and Kochine, I. N., Basic Concepts in the Theory of Seepage of Homogeneous Liquids in Fissured Rocks. J. App. Math., 24, pp. 1286-1303, 1960.

[2] Warren, J. E. and Root, P. J., The Behaviour of Naturally Fractured Reservoirs. SPEJ, pp. 245-255, Sep.1963.

[3] Chen Huan-Zhang, Numerical simulation of conning behavior of a single well in a naturally fractured reservoir. SPEJ, pp. 879-886, Dec. 1983.

[4] Kazemi, H., Merrill, L. S. Jr., Porterfield, K. L., and Zeman, P. R., Numerical simulation of water-oil flow in naturally fractured reservoirs. SPEJ, 16 (6), pp. 318-326, 1976.

[5] Gilman J. R. and Kazemi H., Improvement in Some Simulation of NFR. SPEJ, pp. 695-707, Aug. 1983.

[6] Thomas, L. K., Dixson, T. N., and Pierson, R. G., Fractured Reservoir Simulation. SPEJ, pp. 42-54, Feb.1983.

[7] Litvak, B. L., Simulation and Characterization of Naturally Fractured Reservoirs, a paper presented at the Reservoir Characterization Technical Conference, Dallas, TX., Apr. 1985. 
[8] Sonier, F., Souillard, P. and Blaskovich, F. T, Numerical Simulation of Naturally Fractured Reservoirs, SPE paper 15627 presented at, the 1986 SPE Annual Technical Conference and Exhibition, New Orleans, LA. Oct. 1986.

[9] Kazemi, H., Gilman, J. R., and Elsharkawy, A. M., Analytical and numerical solution of oil recovery from fractured reservoirs using empirical transfer function. SPE Reserv. Eng., 7(2), pp. 219-227, 1992.

[10] Coats, K. H., Implicit Compositional Simulation of Single-Porosity and Dual-Porosity Reservoirs. Proc. of the SPE Symposium on Reservoir Simulation in Houston, TX, Feb. 6-8, SPE paper 18427, 1989.

[11] Lim, K. T. and Aziz, K., Matrix Fracture Transfer Shape Factor for Dual Porosity Simulators. Journal of Petroleum Science and Engineering, 13, pp. 169-178, 1995.

[12] Yu-Shu, Lehua Pan, and Karsten Pruess, A physically based approach for modeling multiphase fracture-matrix interaction in fractured porous media. Advances in Water Resources, 27, pp. 875-887, 2004.

[13] Zoltan E. Heinemann, and Georg M. Mittermeir, Derivation of the KazemiGilman-Alsharkawy generalized dual porosity shape factor. Transp Porous Med, 91, pp. 123-132, 2012.

[14] Chang M., Deriving the shape factor of a fractured rock matrix,” Technical report, NIPER, Bartlesville, Oklahoma, Sep. 1993.

[15] Pallav Sarma., New Transfer Functions for Simulation of Naturally Fractured Reservoirs with Dual Porosity Model. MSc Thesis, Stanford University, May.2003.

[16] Pallav Sarma and Khalid Aziz., New Transfer Functions for Simulation of Naturally Fractured Reservoirs with Dual Porosity Model. SPE Journal, pp. 328-340, Sep. 2006.

[17] Rangel-German, E. R. and Kovscek, A. R., Experimental and analytical study of multidimensional imbibition in fractured porous media. Journal of Petroleum Science and Engineering, 36, pp. 45-60, 2002.

[18] Rangel-German, E. R. and Kovscek, A. R., Time-dependent matrix-fracture shape factor for partially and completely immersed fracture. Journal of Petroleum Science and Engineering, 54, pp. 149-163, 2006.

[19] Rangel-German, E. R., Kovscek, A. R., and Akin S., Time-dependent matrix-fracture shape factor for uniform and non-uniform pressure boundary conditions. Transp Porous Med, 83, pp. 591-601, 2010.

[20] Hulyun Lu, Ginevra Di Donato and Martin J. Blunt, General transfer functions for multiphase flow in fractured reservoirs. SPE Journal, pp. 289297, Sep. 2008.

[21] Hassan Hassanzadeh, Mehran Pooladi-Darvish, Shahram Atabay, Shape factor in the drawdown solution for well testing of dual-porosity systems. Advances in Water Resources, 32, pp. 1652-1663, 2009.

[22] Hassan Golghanddashti, A new analytically derived shape factor for gas-oil gravity drainage mechanism. Journal of Petroleum Science and Engineering, 77, pp. 18-26, 2011. 
[23] Ehsan Ranjbar and Hassan Hassanzadeh, Matrix-Fracture transfer shape factor for modeling flow of a compressible fluid in dual-porosity media. Advances in Water Resources, 34, pp. 627-639, 2011.

[24] Ehsan Ranjbar, Hassan Hassanzadeh, Zhangxin Chen, Effect of fracture pressure depletion regimes on the dual-porosity shape factor for flow of compressible fluids in fractured porous media. Advances in Water Resources, 34, pp. 1681-1693, 2011. 